\title{
Erratum: Density functional theory study of $\mathrm{La}_{2} \mathrm{Ce}_{2} \mathrm{O}_{7}$ : Disordered fluorite versus pyrochlore structure [Phys. Rev. B 84, 054110 (2011)]
}

D. E. P. Vanpoucke, P. Bultinck, S. Cottenier, V. Van Speybroeck, and I. Van Driessche

(Received 11 February 2015; published 19 February 2015)

DOI: 10.1103/PhysRevB.91.059902

PACS number(s): 61.66.-f, 61.50.-f, 61.05.cp, 99.10.Cd

The inset of Fig. 3 of our paper erroneously indicates the first prototypical pyrochlore reflection as (331) instead of (311). In Table II, the PBE $+U$ vacancy formation energy $E_{\mathrm{vac}, f}$ of the SQS should be $-0.091 \mathrm{eV}$ instead of $-0.91 \mathrm{eV}$, as can be deduced from the heat of formation $\Delta H_{f}$ of the SQS NV and SQS systems. The remaining content of the paper and all conclusions remain unaffected. 